\title{
Stimuli-Responsive Smart Polymers and Structures: Characteristics and Applications
}

\author{
Junshi Zhang $\mathbb{D},{ }^{1}$ Gianluca Rizzello, ${ }^{2}$ Jianwen Zhao, ${ }^{3}$ Qi Shen, ${ }^{4}$ and Yanjie Wang $\mathbb{D}^{5}$ \\ ${ }^{1}$ Department of Mechanical and Biomedical Engineering, City University of Hong Kong, Kowloon, Hong Kong \\ ${ }^{2}$ Department of Systems Engineering, University of Saarland, Saarbrücken, Germany \\ ${ }^{3}$ Department of Mechanical Engineering, Harbin Institute of Technology, Weihai, China \\ ${ }^{4}$ Active Materials and Smart Living Laboratory, University of Nevada, Las Vegas, Las Vegas, NV, USA \\ ${ }^{5}$ School of Mechanical and Electrical Engineering, Hohai University, Changzhou, China \\ Correspondence should be addressed to Junshi Zhang; jzhan26@cityu.edu.hk
}

Received 30 May 2018; Accepted 30 May 2018; Published 4 July 2018

Copyright (c) 2018 Junshi Zhang et al. This is an open access article distributed under the Creative Commons Attribution License, which permits unrestricted use, distribution, and reproduction in any medium, provided the original work is properly cited.

The functional polymers and structures can be actuated by the external stimulation, including the light, temperature, and electric field. Nowadays, various smart polymers are studied as soft sensors and actuators or are designed to compose the smart structures to own required performances. The typical soft smart polymers involve dielectric elastomers, ionic-polymer metal composites, hydrogel, shape memory polymers, and so on. During recent years, research on the stimuli-responsive polymers and structures has been extensively performed, especially the electro-responsive soft materials. This special issue is aimed at creating a multidisciplinary forum of discussion on the most recent research advances in synthesis, characterization, design, and application of soft smart polymer and structures, providing a potential guided outlook for future studies.

The knowledge and understanding of such functional materials are of great importance for our practical living. In this special issue, some investigations related to stimuliresponsive smart materials are shown, including different topics, such as dielectric elastomer, hydrogel, and shape memory polymer. In the paper "Dynamic Electromechanical Response of a Viscoelastic Dielectric Elastomer under Cycle Electric Loads," J. Sheng and Y. Zhang explored the nonlinear dynamic performance of the viscoelastic dielectric elastomer when the AC voltage is applied. In this study, a thermodynamic model is set up to characterize the influence of viscoelasticity on the electromechanical and dynamic response of a viscoelastic DE. The time-dependent dynamic deformation, the hysteresis, and the dynamic stability undergoing viscoelastic dissipative processes are investigated. In the paper "Stimuli-Responsive Hydrogels Based on Polyglycerol Crosslinked with Citric and Fatty Acids," three hydrogels were studied with varying molar ratios of a crosslinking agent. It was found that crosslink amount, type, and size play a crucial role in swelling, thermal, mechanical, and stimuli-responsive properties. In the paper "Experimental and Numerical Study of the Interfacial Shear Strength in Carbon Fiber/Epoxy Resin Composite under Thermal Loads," H. Wang et al. examined the influence mechanism of temperature on the interfacial shear strength between carbon fiber and epoxy resin matrices under various thermal loads using experimental and numerical simulation methods. To evaluate the change in IFSS as a function of the increase in temperature, a microbond test was performed under a controlled temperature environment from $23^{\circ} \mathrm{C}$ to $150^{\circ} \mathrm{C}$. In the paper "The Influence of Water and Solvent Uptake on Functional Properties of Shape-Memory Polymers," E. Ghobadi et al. have investigated the effects of different solvents (i.e., water, acetone, and ethanol) on the functional properties of SMP, by means of both experiments and numerical simulations. The aim of the proposed study is to understand whether targeted physical aging "prior to programming" can influence the functional properties of a commercially available SMP (Estane, Lubrizol, Oevel, Westerlo, Belgium). In the paper "Comparison of CMM and MicroCT Volumetric Analysis of Polyethylene Tibial Knee Inserts 
in Total Knee Replacement," W. Jiang et al. investigated the ultrahigh molecular weight polyethylene (UHMWPE) bearings, which are used for orthopaedic joint replacement. CMM and micro-CT measurement techniques are used for volumetric loss with gravimetric measurement as the reference. The comparison results indicate that gravimetric measurement remains the gold standard and the CMM measurement took less time and had better precision, accuracy, and repeatability compared to the micro-CT measurement technique.

\section{Acknowledgments}

We thank all the authors for their invaluable contributions and the reviewers for taking their invaluable time to review for this special issue.

Junshi Zhang

Gianluca Rizzello

Jianwen Zhao

Qi Shen

Yanjie Wang 


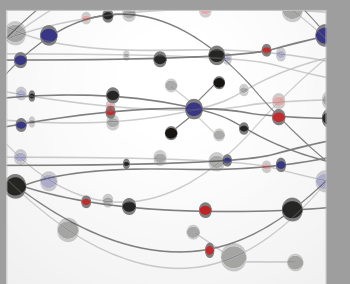

The Scientific World Journal
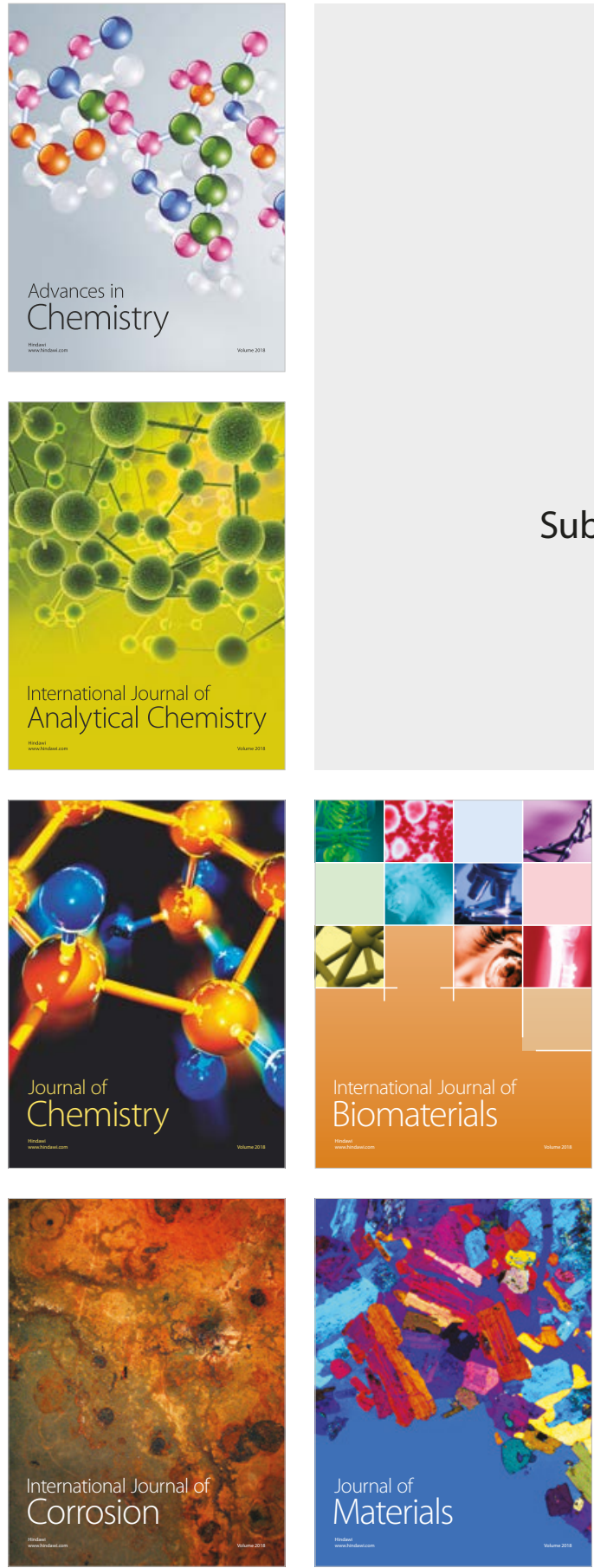

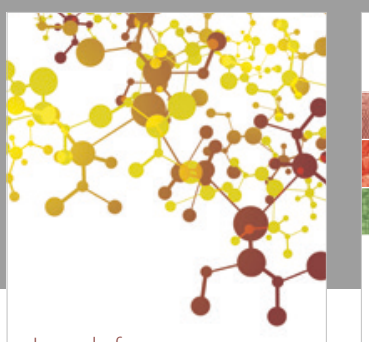

Journal of

Applied Chemistry
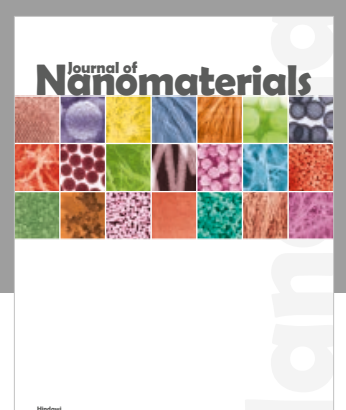

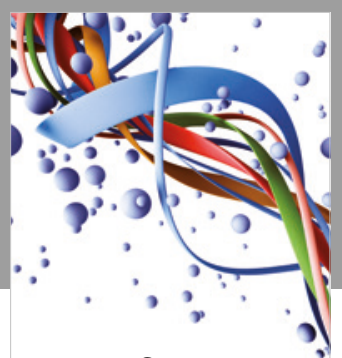

Scientifica

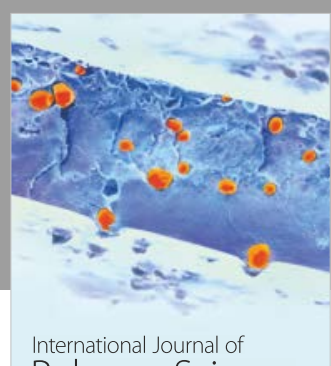

Polymer Science

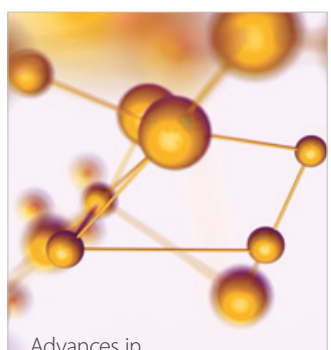

Physical Chemistry
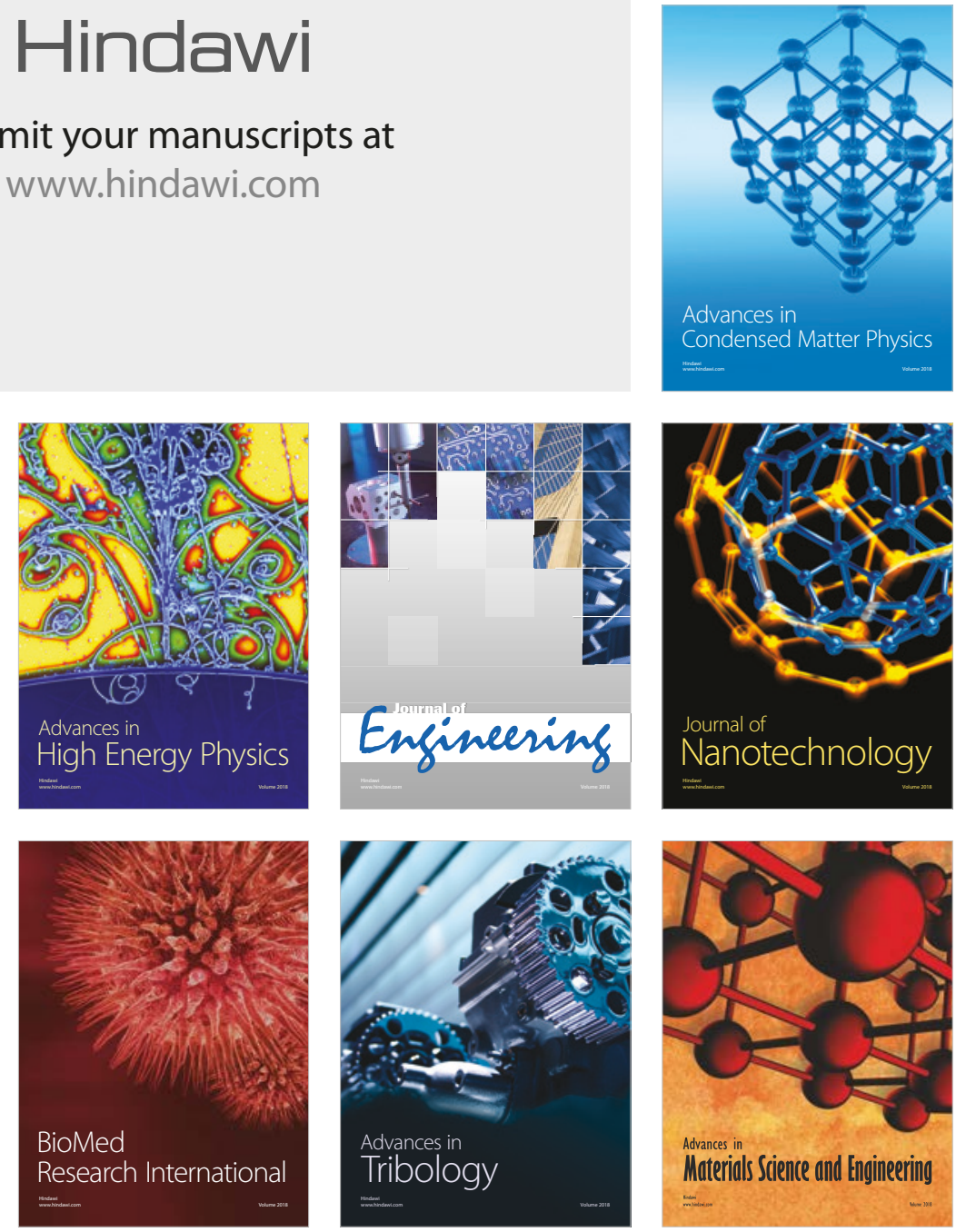